\title{
Effect of precision antiplatelet therapy based on CYP2C19/11-dhTxB2 variability in prognosis of ischemic stroke/TIA patients: study protocol for a multicenter randomized controlled trial
}

\author{
JiaJing Wang \\ Jianglong Tu \\ Jie kuang \\ Nanchang University \\ Katherine Starcevich \\ University of Nevada \\ Yanqiu Ge \\ Nanchang University \\ Chen Peng \\ Nanchang University \\ Yingping Yi (D 13807089678@126.com ) \\ Nanchang University \\ Shujuan Yin \\ Nanchang University \\ Xiaolin Zhang \\ Nanchang University \\ Jibiao Chen \\ Nanchang University
}

Nanchang University https://orcid.org/0000-0001-9751-2376

The Second Affiliated Hospital of Nanchang University

Study protocol

Keywords: Ischemic stroke, Transient ischemic attack, Precision antiplatelet therapy, Prognosis, Randomized controlled trial

Posted Date: July 8th, 2020

DOl: https://doi.org/10.21203/rs.3.rs-21845/v2 
License: (c) (i) This work is licensed under a Creative Commons Attribution 4.0 International License. Read Full License 


\section{Abstract}

Background: Clopidogrel and aspirin are conventional drugs for treating ischemic stroke (IS) and transient ischemic attack (TIA). However, with the increase of clinical application, many patients have shown clopidogrel resistance and/or aspirin resistance. clopidogrel resistance is related with cytochrome P450-2C19 (CYP2C19) poly m orphism, and aspirin resistance is related to urine concentration of 11dehydroxetane B2. At present, the effect of precision antiplatelet medication based on the cytochrome CYP2C19 genotype test and the 11-dhTxB2 test has not been evaluated prospectively in a large sample.

Methods: This is a randomized controlled trial evaluating the effects of precision antiplatelet medication based on the CYP2C19 genotype test and the 11-dhTxB2 test on IS/TIA patients over 12 months. Outcomes of interest including stroke recurrence, neurologic disabilities defined by the Modified Rankin Scale (mRS), bleeding events, other adverse events, and all-cause mortality will be assessed at the 1st, 3rd, 6th and 12th-month post-discharge. Demographics, risk factors, laboratory investigations, medications, physiological tests, and brain imaging will also be assessed.

Discussion: Some stroke patients have resistance to clopidogrel or aspirin, but there is still no personalized medicine. Our study will conduct free antiplatelet resistance tests and individualized antiplatelet medication for patients in the intervention group, ultimately evaluating individualized medication effectiveness through a one-year follow-up. The research results will help to assess the impact of personalized antiplatelet drug therapy on the prognosis of stroke, thus providing a reference for precise clinical treatment.

Trial registration: Chinese Clinical Trial Registry, ChiCTR1900026492. Registered on 12 October 2019.

\section{Introduction}

Stroke is a leading cause of disability and mortality, affecting 200 per 100,000 people globally ${ }^{[1]}$. The incidence of stroke in China is 354 per 100,000 person-years ${ }^{[2]}$. Acute ischemic stroke is the most common stroke, accounting for $60 \%-80 \%$ of all strokes ${ }^{[3]}$. Clopidogrel and aspirin are recommended for the antiplatelet therapy of IS/TIA patients by the American Heart Association/American Stroke Association guidelines ${ }^{[4,5]}$.

Clopidogrel is a common antiplatelet aggregation drug in clinical practice and plays an important role in treating of $I^{[6]}$. However, with the increase of clinical application, the efficacy of clopidogrel varies with some patients displaying resistance ${ }^{[7-10]}$. Studies have shown that the CYP2C19 gene polymorphism is closely related to the inhibition of clopidogrel on platelets aggregation. This has been used to categorize patients by CYP2C19 metabolizer status based on $* 2, * 3, * 17$ genotypes: poor metabolizers; intermediate metabolizers; extensive metabolizers; ultra-metabolizers ${ }^{[11,12]}$. A genetically tailored antiplatelet therapy within the study population is needed as previous studies have found a high prevalence of clopidogrel resistance in Asian populations ${ }^{[13,14]}$. In recent years, the most common CYP2C19*2, $* 3$ mutation sites in 
the human cytochrome P450 allele have attracted much attention ${ }^{[15-18]}$. Aspirin is widely used in secondary prevention of stroke. However, it is reported that $2 \%$ to $57 \%$ of patients have aspirin resistance ${ }^{[19]}$. In recent years, the relationship between aspirin resistance and stroke recurrence has attracted increasing attention and related research ${ }^{[20]}$.

Wang et al. found that compared to aspirin-only, the use of clopidogrel plus aspirin reduced the risk of a new stroke only in the subgroup of patients who were not carriers of the CYP2C19 loss of-function alleles $^{[21]}$. The impact of CYP2C19 polymorphisms on clopidogrel pharmacodynamics and clinical outcomes (stroke recurrence, composite vascular events, bleeding) of stroke or TIA patients has been well established $^{[22-24]}$. However, the effect of aspirin resistance was not included in previous studies. At present, there is no research reported on genetics tailored therapy guidelines for patients with clopidogrel resistance and/or aspirin resistance.

\section{Objectives}

This multicenter randomized controlled trial aims to investigate the effect of precise antiplatelet drug treatment based on CYP2C19 genotyping and 11-dhTxB2 testing in patients with ischemic stroke/TIA, and to build an intelligent decision-making platform to provide a reference for clinical treatment.

\section{Methods}

\section{Trial design}

This study is a multicentre, large sample, randomized controlled study with a 20 months study case recruitment, and an one-year post enrollment follow-up. Written informed consent is required for all participants. The trial is registered in the Chinese Clinical Trial Registry (ChiCTR1900026492).

The study is being conducted at 14 comprehensive first-class hospitals in Jiangxi Province. The participants are voluntary and randomly assigned to the control and intervention groups. Patients in the intervention group are tested for CYP2C19 genotypes and urine 11-dhTxB2. Finally, according to the test results, individualized medications of clopidogrel or aspirin are developed for each patient in the intervention group. We will not intervene in the clinical medication of patients in the control group. Doctors will prescribe antiplatelet medication based on the patient's condition and existing guidelines.

\section{Participants and recruitment}

Inpatients are screen by a researcher in the neurology department of the participating hospital. A researcher will determine eligible patients then explain the study in detail. Patients who voluntarily 
participate must sign the informed consent form and are asked to leave one to two telephone numbers for contact during the one-year follow-up. The researcher will record all interview information for the research participant. The algorithm for the study schedule/recruitment process is showed in Figure 1.

\section{Eligibility criteria}

Patient eligibility criteria include

(1) age 18 to 85 years,

(2) diagnosis of ischemic stroke (atherosclerotic cerebral infarction) or transient ischemic attack (diagnosis meets the criteria established by the Cerebrovascular Diseases Group of the Chinese Medical Association Neurology Society in 2014) ${ }^{[25]}$,

(3) acute period of onset ( 2 weeks post-event),

(4) NIHSS score $\leq 15$ points,

(5) voluntarily participation and informed consent.

Patient exclusion criteria include

(1) any cancer diagnosis,

(2) cardiac infarction, cerebral infarction with small vessel disease, other causes of cerebral infarction or unexplained cerebral infarction,

(3) stomach disease judged by doctor to be at risk of bleeding,

(4) alteplase thrombolytic usage, as well as some cases requiring anticoagulation or dual antiplatelet $(\mathrm{PCl})$,

(5) hemorrhagic stroke, mixed stroke, or tumor stroke,

(6) whole blood or platelet transfusion in the past two weeks, coagulopathy or other blood diseases,

(7) renal dysfunction (24-hour urine volume $<1000 \mathrm{ml}$, serum creatinine $>180 \mathrm{MMOL} / \mathrm{L}$, urine creatinine $<5 \mathrm{mmol} / \mathrm{L})$,

(8) patients are combined with serious heart, lung and liver system diseases,

(9) poor adherence or inability to complete long-term follow-up, 
(10) current involvement in any clinical trial of research drugs or medical devices (Figure 2).

Patients will be excluded after enrollment if

(1) they are misdiagnosed or misrepresented cases that do not meet the inclusion criteria and meet the exclusion criteria,

(2) they voluntarily withdraw from the study,

(3) the investigator determines that an effective clinical observer can not be continued for medical or other reasons,

(4) they switched to other antiplatelet drugs, or stopped taking antiplatelet drugs, or switched to anticoagulant in the course of the study,

(5) they needed blood/platelets transfusion during the 1-year follow up.

\section{Randomization}

Once eligible patients voluntarily sign the informed consent form, researchers will register each patient in the queue, and perform simple randomization. The ratio of the control group to the intervention group is 1:1, and a random seed number is adopted. We have designed an automatic random grouping program in the Electronic Data Capture (EDC) platform, used by researchers in cooperative hospitals to log in to the platform and implement simple random grouping.

\section{Participant timeline}

Baseline data such as BMI, NIHSS, mRS, imaging examination, and laboratory test results are collected during hospitalization. At discharge, besides individualized drug treatment and medical expenses, the NIHSS and mRS scores for each participant are also evaluated and recorded. All patients are required to follow up at the first, third, sixth, and twelfth months after discharge, and report drug compliance and prognosis (including stroke recurrence, adverse events, mRS, etc.). Table 1 details the participants' schedule of this study. 


\section{CYP2C19 Genotype and 11-dhTxB2 tests}

Three single-nucleotide polymorphisms (SNPs) for CYP2C19 (National Center for Biotechnology Information [NCBI] Genome build 37.1, GenBank NG_008384), including CYP2C19*2 (681G>A, dbSNP rs4244285), CYP2C19*3 (636G>A, dbSNP rs4986893), and CYP2C19*17 (-806C>T, dbSNP rs12248560) [26-29] will be genotyped in participants of intervention group. Genotyping of the 3 SNPs is performed using the PCR+MassARRAY (flight mass spectrometry). Individuals with complete information for each of the 3 SNPs will be included in the current analyses. Patients are categorized by CYP2C19 metabolizer status based on $* 2, * 3$, and $* 17$ genotypes using the common consensus star allele nomenclature.

CYP2C19*2, $* 3$ are loss-of-function alleles, and CYP2C19*17 is gain-of-function allele $e^{[30]}$. Patients with two loss-of-function alleles $(* 2 / * 2, * 2 / * 3$, or $* 3 / * 3$ ) are classified as poor metabolizers, those with one loss-of-function allele $(* 1 / * 2, * 1 / * 3, * 2 / * 17$ or $* 3 / * 17)$ are classified as intermediate metabolizers, and those without loss-of-function allele $\left({ }^{*} 1 /{ }^{*} 1\right)$ are classified as extensive metabolizers ${ }^{[31]}$. Patients carrying $\star 1$ and $* 17$ alleles $\left({ }^{*} 1 / * 17\right.$ or $\left.{ }^{*} 17 / * 17\right)$ are classified as ultra-metabolizers ${ }^{[32]}$.

Aspirin is widely used in secondary prevention of stroke because it inhibits cyclooxygenase (cox) and thus reduces the production of thromboxane A (TXA2), which reflects the activation state of platelets and the function of aspirin in vivo. In this study, aspirin resistance is tested for using 11-dhTxB2 concentration in urine with 11-dhTxB2/urinary creatinine $\geq 1500 \mathrm{pg} / \mathrm{mg}$ used to characterize the presence of aspirin resistance ${ }^{[33,34]}$. Once obtained, results of gene and urine tests will be used to develop personalized drug treatment programs.

\section{Treatments}

On days 1-8, 3-5 $\mathrm{ml}$ whole blood samples and 3-10 ml urine samples for the CYP2C19 genotype and 11dhTxB2 test are taken from patients in the intervention group. The results are usually obtained on day 7-9. After the tests, doctors will adjust the antiplatelet medication according to the results. If the patient is only resistant aspirin, then only clopidogrel is prescribed; if the patient is only resistant to clopidogrel, then only aspirin is prescribed. If the patient is not resistance to aspirin and clopidogrel, we will not change the treatment plan of the patient. If the patient has dual resistance to clopidogrel and aspirin, because of the risk of bleeding, we do not make medication interventions and only recommend that the doctor to increase the dose or use alternative drugs. Patients in the control group receive neither genetic nor antiplatelet testing. This study is single blind to patients. Patients may experience discomfort while having blood drawn for blood samples, and it is an expected harm in this study. If adverse events occur during follow-up, researchers will record, report, and deal with these events. Treatment measures include 
sending patients to a nearby hospital as soon as possible, and/or terminating personalized medication in case of severe bleeding.

\section{Endpoints}

The primary outcome of interest is the recurrence of stroke. Secondary outcomes include bleeding events, $\mathrm{mRS}$, adverse medical reaction and all-cause mortality. Adverse medical conditions include symptoms (nausea, chest pain, etc.), signs (tachycardia, tachycardia, etc.), or abnormal test results (laboratory test results, electrocardiogram, etc.).

\section{Clinical assessment}

Baseline assessment includes: (1) demographics (age, sex, nation, address, vital sign, average annual income, educational level, work, medical payment method); (2) risk factors (smoking, alcohol, hypertension, diabetes, heart diseases, hyperlipemia, dementia, hypohepatia, renal insufficiency, obesity, stroke/malignancy tumor/trauma/

surgical history, r-tPA intravenous thrombolysis); (3) family/personal medical history; (4) medications; (5) laboratory results (blood, urine, biomarkers); (6) physiological tests (BMI, blood pressure, pulse, respiratory rate, cardioechography); (7) brain imaging (MRI, CT, CTA/MRA/DSA); (8) comprehensive neurological assessment (TOAST subtypes, NIHSS, mRS, GCS); (9) symptoms and duration of hospitalization.

\section{Sample size}

In Wang et al.'s study, $58.8 \%$ of participants were carriers of CYP2C19 loss-of-function alleles. Stroke recurrence in non-carriers only: $6.7 \%$ in treatment (clopidogrel plus aspirin) vs. $12.4 \%$ control (aspirinonly). According to the above results, each group needs 417 cases, for a total of 834 . We assume a loss to follow-up rate of $15 \%$ and a design effect of $1.25^{[34]}$. Finally, we calculated a sample size of 1199 , which is about 1200 . We aim to recruit 1,200 to 6,000 patients. If the sample size is not completed within 20 months, we will extend the study time appropriately. 


\section{Data collection and management}

According to the requirements of the project, we designed the data collection table on the EDC platform, defined the research process, the name of the data table, the collected data items, and formed the corresponding data collection guide. Finally, eCRF is constructed based on the research of medical records. Once all participants have completed the trial, all medical records will be entered into the system. After the primary investigator, sponsor, statistical analyst, and data administrators confirm that the data in the database is correct, the data administrators will check the integrity of the data. Finally, all the data will be locked, and the data administrator will import it into the designated database and send it to the statisticians for statistical analysis. The locked data can no longer be edited, and the problems found after the data locking can be corrected in the statistical analysis program after confirmation. After the trial is over, the data administrator will put forward a request for closing EDC and cancel all account access rights after obtaining the permission of the sponsor. Finally, when the data is completely backed up, the EDC will be shut down.

\section{Quality control measures}

This study consists of sponsor commissions and contract research organization to complete the audit work, to ensure that the rights and interests of the subjects in the clinical trials are guaranteed, the recorded data are accurate and complete, and to ensure the test follows the follows the approved program, ¿Quality Management Standards for Drug Clinical Trials $\square^{[35]}$ and related regulations.

If there are problems during the actual implementation of the clinical trial, it is necessary to revise the plan and submit it to the sponsor. After consultation and discussion by the multi-center coordination committee, the clinical research unit will revise the plan and then submit to the sponsor and the participating units for signature approval. Finally, it is submitted to the ethics committee for approval and implementation. If important new information related to the test drug is found, the informed consent form must be modified in writing form and sent to the Ethics Committee for approval.

\section{Statistical analysis}

Continuous variables that conform to the normal distribution are described using the mean (standard deviation). Those that do not conform to the normal distribution are presented as the median (inter- 
quartile range). Categorical variables are expressed as the number (percentage). Pearson's $\chi 2$ test of independence will be used for categorical variables, and the analysis of variance and Mann-Whitney $\mathrm{U}$ test will be used for numerical variables, as appropriate. Multiple imputation will be used to impute missing values, under a missing at random assumption, so as to reduce bias and avoid excluding participants from the analysis. Comparison of endpoint incidence between intervention and control groups will be assessed by risk rate (RR). Multivariate Cox regression analysis will be used for the time-toevent data with adjustment of known confounders. In this study, the estimates of missing values for the primary variables are carried-forward to the absence of test data using the results of the closest observation. All statistical tests will be two-sided, and $\mathrm{P}<0.05$ is considered statistically significant. Statistical analysis will be performed via SAS statistical software, version 9.3 (SAS Institute Inc.).

\section{Discussion}

Stroke is the second most common cause of death and the leading cause of long-term disability worldwide ${ }^{[36]}$. Clopidogrel and aspirin are conventional drugs for the treatment of IS and TIA patients ${ }^{[37]}$, but a large number of patients have a genetic predisposition to clopidogrel resistance and/or aspirin resistance ${ }^{[38,39]}$. The effects of individualized medication based on CYP2C19 genotype test and 11dhTxB2 test are still unknown. Currently, there is a lack of large-scale study for the evaluation of the clinically accurate dual antiplatelet therapy standards based on genetic and drug metabolite tests.

The CYP2C19 genotype test is expensive and not widely available in Chinese hospitals. Our study collects financial information such as family annual income, hospitalization expenses, and drug costs for the enrolled patients. Using this information, we will conduct economic analysis to evaluate the long-term benefits of CYP2C19 genotype/11-dhTxB2 tests for IS/TIA patients, to provide evidence for a general genotype /11-dhTxB2 test..

Our proposed study has several strengths. First, this is the first study to assess the effect of individualized medication based on the CYP2C19 genotype test and 11-dhTXB2 test for IS/TIA patients. Additionally, this is a multicentre prospective cohort study with multilateral evaluations and analyses of gene polymorphism, and prognosis of IS/TIA.

There are several potential limitations. First, no prescribing guidelines standards for patients resistant to both clopidogrel and aspirin currently exist. Since forcibly increasing antiplatelet dosage may lead to a higher risk of bleeding, this study did not adjust the medication for this group of patients, but only provided a reference for clinicians. Second, there is still the possibility of unrecognised confounders despite the multicentre design. Inconsistent levels of investigators and patient medication compliance in different hospitals may affect the effectiveness of individualized medicine.

Developing personalized antiplatelet medications and reducing the recurrence rate of stroke is an urgent problem in Chinese society. We hope that our study will contribute to a better understanding of the 
association between individualized antiplatelet medications and stroke prognosis, and develop a standardized stratified treatment plan for IS/TIA patients.

In conclusion, this study will evaluate the effect of individualized medications on the prognosis of stroke/transient ischemic attack and provide a reference for clinical medications. The results of this study, whether positive or negative, will be of great interest to both IS/TIA clinicians and patients.

\section{Trial status}

At the time of manuscript submission, recruitment for this study is ongoing. The recruitment of patients began in June 2019 and will continue until December 2020. Participants will be followed for one-year and the study will end in December 2021.

\section{List Of Abbreviations}

IS: ischemic stroke; TIA: transient ischemic attack; 11-dhTxB2: 11-dehydroxetane B2; CYP2C19: cytochrome P450 2C19; mRS: Modified Rankin Scale; PM: poor metabolizers; IM: intermediate metabolizers; EM: extensive metabolizers; UM: ultra-metabolizers; NIHSS: National Institute of Health stroke scale; PCl: percutaneous coronary intervention; BMl: Body Mass Index; NCBI: National Center for Biotechnology Information; cox: cyclooxygenase; TXA2: thromboxane A; TOAST: Trial of Org 10172 in acute stroke treatment; EDC: Electronic Data Capture; eCRF: Electronic case report form; SD: standard deviation; IQR: interquartile range; RR: risk rate.

\section{Declarations}

\section{Ethics approval and consent to participate}

This study received ethical approval from the Medical Ethics Committee of the Second Affiliated Hospital of Nanchang University: (2018) Medical Research Review No.05. Informed consent is obtained from all study participants. All patients voluntarily sign the informed consent form, indicating that they agree to participate.

\section{Consent for publication}

Not applicable.

\section{Availability of data and materials}


The datasets used and analyzed during the current study are available

from the corresponding author on reasonable request. The consent form is

available from the corresponding author on request.

\section{Competing interests}

On behalf of all authors, the corresponding author states that there is no conflict of interest.

\section{Funding}

This work is supported by the National Key R\&D Program of China (2018YFC1312902), Science and Technology Innovation Platform of Jiangxi Department of Science and Technology (20171BCD40024) and Jiangxi Provincial Key R\&D Program Class B Project (20181ACH80004).

\section{Authors' contributions}

JJW: drafting and revising the protocol. YPY, JK: principle investigator and contributed to the concept and design of the protocol. JLT, SK, YQG, CP, SJY, XLZ and JBC: critical revising of the protocol. All authors read and approved the final version of this manuscript.

\section{Acknowledgements}

We thank Lijun Xu for his input to parts of the study design, and Lu Shi, Zhaohui Zhang, Yitong He, Linli Lu, Jianwei Luo for helping with the one-year follow-up. We would like to thank these doctors for their contributions in organization, recruitment, data collection, sample collection, and medication adjusting: Daojun Hong, Ping Fan, Dan Ding, Xiangjun Zeng, Qianxi Chen, Zhihong Huang, Xing Huang, Fangmei Chen, Zefei Lai, Ying Zeng, Yumei Li.

\section{Author details}

${ }^{1}$ Jiangxi Provincial Key Laboratory of Preventive Medicine, School of Public Health, Nanchang University, Nanchang, Jiangxi Province, China. ${ }^{2}$ Department of Information, The Second Affiliated Hospital of Nanchang University, Nanchang, Jiangxi Province, China. ${ }^{3}$ Department of neurology, The Second 
Affiliated Hospital of Nanchang University, Nanchang, Jiangxi Province, China. ${ }^{4}$ School of Community Health Sciences, University of Nevada, Reno, Nevada, USA.

\section{References}

1. Benjamin EJ, Blaha MJ, Chiuve SE, et al. Heart Disease and Stroke Statistics-2017 Update: A Report From the American Heart Association. Circulation. 2017; 135(10): e146-e603.

2. GBD 2016 Stroke Collaborators. Global, regional, and national burden of stroke, 1990-2016: a systematic analysis for the Global Burden of Disease Study 2016. Lancet Neurol. 2019;18(5):439458.

3. Zhao D, Liu J, Wang W, et al. Epidemiological transition of stroke in China: 21-year observational study from the Sino-MONICA-Beijing Project. Stroke. 2008;39(6):1668-1674.

4. Powers WJ, Rabinstein AA, Ackerson T, et al. 2018 guidelines for the early management of patients with acute ischemic stroke: a guideline for healthcare professionals from the American Heart Association/- American Stroke Association. Stroke. 2018(49):e46-e110.

5. Kernan WN, Ovbiagele B, Black HR, et al. Guidelines for the prevention of stroke in patients with stroke and transient ischemic attack: a guideline for healthcare professionals from the American Heart Association/American Stroke Association. Stroke. 2014(45):2160-2236.

6. Xu J, Wang A, Wangqin R, Mo J, Chen Z, Dai L, Meng X, Zhao X, Wang Y, Li H4, Chen W, Xian Y, Wang $\mathrm{Y}, \mathrm{CHANCE}$ investigators. Efficacy of clopidogrel for stroke depends on CYP2C19 genotype and risk profile. Ann Neurol.2019; 86(3):419-426

7. Kernan WN, Ovbiagele B, Black HR, et al. Guidelines for the prevention of stroke in patients with stroke and transient ischemic attack: a guideline for healthcare professionals from the American Heart Association/American Stroke Association. Stroke. 2014; 45(7):2160-2236.

8. Zhao D, Liu J, Wang W, Zeng Z, Cheng J, Liu J, Sun J, Wu Z, Epidemiological transition of stroke in China: twenty-one-year observational study from the Sino-MONICA-Beijing Project. Stroke. 2008 (39):1668-1674.

9. Yi X, Han Z, Zhou Q, Lin J, Liu P, 20-hydroxyeicosatetraenoic acid as a predictor of neurological deterioration in acute minor ischemic stroke. Stroke. 2016(47): 3045-3047.

10. Spokoyny I, Barazangi N, Jaramillo V, Rose J, Chen C, Wong C, Tong D, Reduced clopidogrel metabolism in a multiethnic population: prevalence and rates of recurrent cerebrovascular events. $\mathrm{J}$ Stroke Cerebrovasc Dis. 2014 (23): 694-698.

11. Hoh BL, Gong Y, McDonough CW, Waters MF, Royster AJ, Sheehan TO, Burkley B, Langaee TY, Mocco J, Zuckerman SL, Mummareddy N, Stephens ML, Ingram C, Shaffer CM, Denny JC, Brilliant MH, Kitchner TE, Linneman JG, Roden DM, Johnson JA, CYP2C19 and CES1 polymorphisms and efficacy of clopidogrel and aspirin dual antiplatelet therapy in patients with symptomatic intracranial atherosclerotic disease. J Neurosurg. 2016(124):1746-1751. 
12. Yilong Wang, Xingquan Zhao, Jinxi Lin, Hao Li, S Claiborne Johnston, Yi Lin, Yuesong Pan, Liping Liu, David Wang, Chunxue Wang, Xia Meng, Jianfeng Xu, Yongjun Wang, CHANCE investigators, Association Between CYP2C19 Loss-of-Function Allele Status and Efficacy of Clopidogrel for Risk Reduction Among Patients With Minor Stroke or Transient Ischemic Attack. JAMA. 2016; 316(1):7078.

13. Kwon HM, Lee YS, Bae HJ, Kang DW. Homocysteine as a predictor of early neurological deterioration in acute ischemic stroke. Stroke. 2014;45(3):871-873.

14. Yi X, Lin J, Wang Y, Zhou Q, Wang C, Cheng W, Chi L, Association of cytochrome P450 genetic variants with clopidogrel resistance and outcomes in acute ischemic stroke. $\mathrm{J}$ Atheroscler Thromb. 2016 (23): 1188-1200.

15. Uematsu T, Takasaki W, Kosuge K, Wada K, Matsuno H, Tanaka Y, Yamamura N, Nakashima M. Changes in plasma and urinary 11-dehydrothromboxane B2 in healthy subjects produced by oral CS518, a novel thromboxane synthase inhibitor. Journal of Clinical Pharmacology.1993;45(3):283-6.

16. Moris D, Bakoyiannis C, Karaolanis G, Georgopoulos S. Reply to effect of CYP2C19*2 and *3 on clinical outcome in ischemic stroke patients treated with Clopidogrel. J Neurol Sci. 2017, 372-464.

17. Zhu WY, Zhao T, Xiong XY, Li J, Wang L, Zhou Y, Gong ZL, Cheng SY, Liu Y, Shuai J, Yang QW, Association of CYP2C19 polymorphisms with the clinical efficacy of clopidogrel therapy in patients undergoing carotid artery stenting in Asia. Sci Rep. 2016 (3): 6-25478.

18. Lin J, Han Z, Wang C, Yi X, Chai Z, Zhou Q, Huang R, Dual therapy with clopidogrel and aspirin prevents early neurological deterioration in ischemic stroke patients carrying CYP2C19 *2 reducedfunction alleles. Eur J Clin Pharmacol. 2018 (74):1131-1140.

19. Grinstein J, Cannon CP. Aspirin resistance: current status and role of tailored therapy. Clin Cardiol. 2012; 35(11):673-681.

20. Ke Xu, Sen Ye, Shuhua Zhang, Mingwen Yang, Tiantian Zhu, Deyu Kong, Jun Chen, Lei Xu, Jimin $\mathrm{Li}$, Hui Zhu, Fei Wang, Lu Yang, Jing Zhang, Yuansheng Fan, Lianghong Ying, Xianqing Hu, Xiaofeng Zhang, Noel C Chan, Chunjian Li, Impact of Platelet Endothelial Aggregation Receptor-1 Genotypes on Platelet Reactivity and Early Cardiovascular Outcomes in Patients Undergoing Percutaneous Coronary Intervention and Treated With Aspirin and Clopidogrel. Circ Cardiovasc Interv. 2019; 12(5):e007019.

21. Yongjun Wang, Yilong Wang, Xingquan Zhao, Liping Liu, David Wang, Chunxue Wang, Chen Wang, Hao Li, Xia Meng, Liying Cui, Jianping Jia, Qiang Dong, Anding Xu, Jinsheng Zeng, Yansheng $\mathrm{Li}$, Zhimin Wang, Haiqin Xia, S Claiborne Johnston, CHANCE Investigators. Clopidogrel with aspirin in acute minor stroke or transient ischemic attack. N Engl J Med. 2013;369(1):11-19.

22. Lewis JP, Backman JD, Reny JL, et al. Pharmacogenomic Polygenic Response Score Predicts Ischemic Events and Cardiovascular Mortality in Clopidogrel-Treated Patients. Eur Heart J Cardiovasc Pharmacother. 2019, Sep 3:pvz045.

23. Xu J, Wang A, Wangqin R, Mo J, Chen Z, Dai L, Meng X, Zhao X, Wang Y, Li H, Chen W, Xian Y, Wang Y, Efficacy of clopidogrel for stroke depends on CYP2C19 genotype and risk profile. Ann Neurol. 
2019;86(3):419-426.

24. Wang T, Pan Y, Lin J, Anand R, Wang D, Johnston SC, Meng X, Li H, Zhao X, Liu L, Wang Y, Wang Y, Influence of smoking on CYP2C19 genetic variants and clopidogrel efficacy in patients with minorstroke or transient ischaemic attack. Eur J Neurol. 2019; 26(9):1175-1182.

25. Chinese Medical Association Neurology Branch, Chinese Medical Association Neurology Branch Cerebrovascular Disease Group. Guidelines for the diagnosis and treatment of acute ischemic stroke in China 2014 (In Chinese). Chinese Journal of Neurology, 2015, 48(4):246-257.

26. Hulot JS, Bura A, Villard E, Azizi M, Remones V, Goyenvalle C, Aiach M, Lechat P, Gaussem P, Cytochrome P4502C19 loss-of-function polymorphisms is a major determinant of clopidogrel responsiveness in healthy subjects. Blood. 2006(108): 2244-2247.

27. Kitzmiller JP, Groen DK, Phelps MA, Sadee W, Pharmacogenomic testing: relevance in medical practice: why drugs work in some patients but not in others. Cleve Clin J Med. 2011(78):243-257.

28. Wang Y, Cai H, Zhou G, Zhang Z, Liu X, Effect of CYP2C19*2 and *3 on clinical outcome in ischemic stroke patients treated with clopidogrel. Journal of the Neurological Sciences. 2016(369):216-219.

29. Chen YB, Zhou ZY, Li GM, Xiao GX, Yu WB, Zhong SL, Cai YF, Jin J, HuangM, Influences of an NR1I2 polymorphisms on heterogeneous antiplatelet reactivity responses to clopidogrel and clinical outcomes in acute ischemic stroke patients. Acta Pharmacologica Sinica. 2019(40):762-768.

30. Jeong TD, Kim SM, Kim HJ, Lee W, Kwon SU, Min WK, Kang DW, Chun S, CYP2C19 genotype and early ischemic lesion recurrence in stroke patients treated with clopidogrel. J Stroke Cerebrovasc Dis. 2015(24):440-446.

31. Yi X, Zhou Q, Wang C, Lin J, Cheng W, Chi L, Concomitant Use of Proton Pump Inhibitors and Clopidogrel Is Not Associated with Adverse Outcomes after Ischemic Stroke in Chinese Population. Stroke Cerebrovasc Dis. 2016(25):2859-2867.

32. Kitazono T, Ikeda Y, Nishikawa M, Yoshiba S, Abe K, Ogawa A. Influence of cytochrome P450 polymorphisms on the antiplatelet effects of prasugrel in patients with non-cardioembolic stroke previously treated with clopidogrel. J Thromb Thrombolysis. 2018; 46(4):488-495.

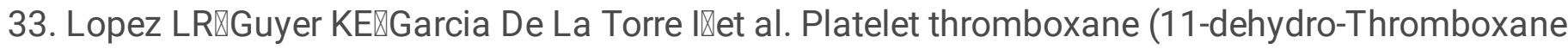
B2) and aspirin response in patients with diabetes and coronary artery disease. World J Diabetes. 2014; 5(2):115-127.

34. Luo Wei. Research of Sample Allocation Based on Stratification Design Effect. Statistics \& Decision. 2017(16): 20-23.

35. Quality Management standard for clinical trials of drugs (In Chinese). Chinese Medical Guide. 2003(05):367-372.

36. GBD 2015 Mortality and Causes of Death Collaborators. Global, regional, and national life expectancy, all-cause mortality, and cause-specific mortality for 249 causes of death, 1980-2015: a systematic analysis for the global burden of disease study 2015. Lancet. 2016(388):1459-1544.

37. Wenshan Sun, Yongkun Li, Junrong Li, Zhizhong Zhang, Wusheng Zhu, Wenhua Liu, Qiankun Cai, Xiaomeng Wang, Liping Cao, Wen Bai, Xinying Fan, Minmin Ma, Ruibing Guo, Xinfeng Liu, Gelin 
$\mathrm{Xu}$, Variant recurrent risk among stroke patients with different CYP2C19 phenotypes and treated with clopidogrel. Platelets. 2015; 26(6):558-562.

38. Kwon HM, Lee YS, Bae HJ, Kang DW, Homocysteine as a predictor of early neurological deterioration in acute ischemic stroke. Stroke. 2014; 45(3):871-873.

39. Vahidy FS, Hicks WJ, Acosta I, Hallevi H, Peng H, Pandurengan R, Gonzales NR, Barreto AD, MartinSchild S, Wu TC, Rahbar MH, Bambhroliya AB, Grotta JC, Savitz SI, Neurofluctuation in patients with subcortical ischemic stroke. Neurology. 2014; 83(5):398-405.

\section{Table}

Table1. Schedule of assessments for the strategy to obtain CYP2C19/11-dhTxB2 tests' effect by evaluating registry study

Study period Baseline assessment $\quad$ Follow-up $\quad$ Follow-up

\begin{tabular}{|c|c|c|c|c|c|c|}
\hline \multicolumn{7}{|c|}{ complete/early termination } \\
\hline Visit & V1 & V2 & V3 & V4 & V5 & V6 \\
\hline Timing & $1 d$ & $7 d$ & $1 \mathrm{~m}( \pm 7 \mathrm{~d})$ & $3 \mathrm{~m}( \pm 7 \mathrm{~d})$ & $6 \mathrm{~m}( \pm 7 \mathrm{~d})$ & $12 \mathrm{~m}( \pm 7 \mathrm{~d})$ \\
\hline Informed consent & 0 & & & & & \\
\hline Screening/eligibility & - & & & & & \\
\hline Basic informations* & - & & & & & \\
\hline Laboratory tests** & 0 & ○ & & & & \\
\hline Physiological tests*** & 0 & ○ & & & & \\
\hline Brain imagings $* * * *$ & 0 & 0 & $\bullet$ & $\bullet$ & 0 & O \\
\hline NIHSS & - & - & & & & \\
\hline $\mathrm{mRS}$ & 0 & 0 & - & 0 & 0 & 0 \\
\hline GCS & - & & & & & \\
\hline CYP2C19 genotype/11-dhTxB2 tests & & 0 & & & & \\
\hline Medications & 0 & 0 & $\bullet$ & 0 & 0 & 0 \\
\hline Clinical outcomes/endpoints $\dagger$ & & & - & - & 0 & - \\
\hline
\end{tabular}

- : required, ๑: optional.

*Basic information include: demographics, risk factors, family/personal medical history, symptoms and duration at admission, etc.

**Laboratory tests include: blood, urine, biomarkers, etc.

***Physiological tests include: BMI, blood pressure, pulse, respiratory rate, cardioechography. 
****Brain imaging include: MRI,CT,CTA/MRA/DSA.

†Clinical outcomes/endpoints include: stroke recurrence, safety/fatal bleeding events, adverse events, neurological impairment and all-cause mortality.

\section{Figures}
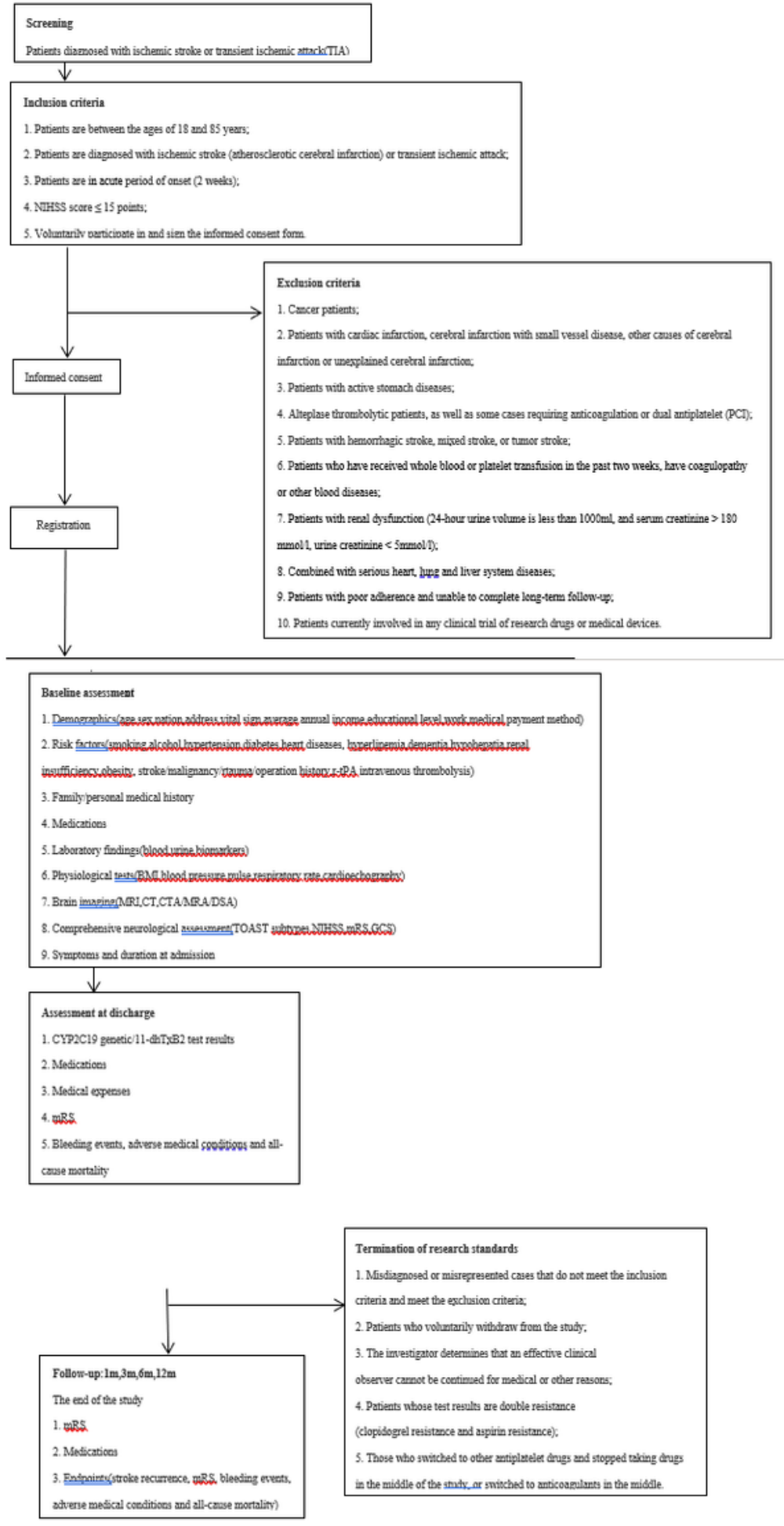
Figure 1

Flow chart of the study design. NIHSS, National Institutes of Health Stroke Scale; mRS, Modified Rankin Scale; r-tPA, recombinant tissue plasminogen activator; BMI, body mass index; MRI, magnetic resonance imaging; CT, computed tomography; CTA, CT angiography; MRA, magnetic resonance angiography; DSA, digital subtraction angiography; TOAST, trial of org 10172 in acute stroke treatment; GCS, Glascow Coma Scale; $m$, month.

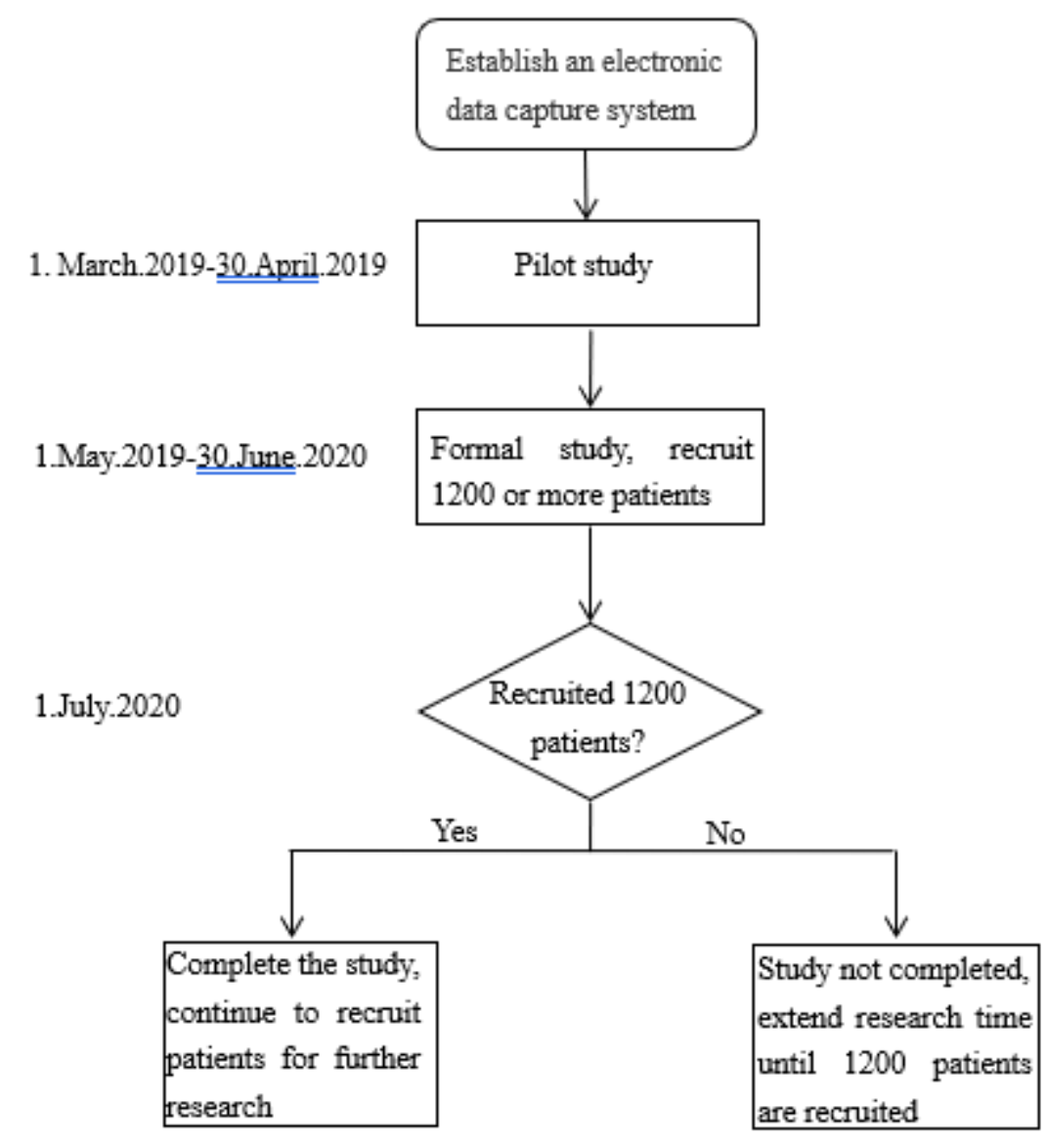

Figure 2

Algorithm about the study timeline/recruitment process.

\section{Supplementary Files}

This is a list of supplementary files associated with this preprint. Click to download.

- SPIRITChecklist.doc 